\title{
Effect of diameter ratio on unsteady flow past two side-by-side cylinders at $\mathrm{Re}=\mathbf{2 0 0 0}$
}

Cite as: AIP Conference Proceedings 2293, 030027 (2020); https://doi.org/10.1063/5.0028770 Published Online: 25 November 2020

R. Rajita Shenoi, Neeraj P. Manelil, T. Sundararajan, and Shaligram Tiwari

\section{ARTICLES YOU MAY BE INTERESTED IN}

Effect of aspect ratio on wake flow characteristics of prolate spheroid at $\mathrm{Re}=200$

AIP Conference Proceedings 2293, 030019 (2020); https: / doi.org/10.1063/5.0027090

Two-dimensional numerical investigation of the cavitation sheet over a NACA 16-012 AIP Conference Proceedings 2293, 030025 (2020); https://doi.org/10.1063/5.0026517

Numerical simulation of gusty flow past cube with wings

AIP Conference Proceedings 2293, 030010 (2020); https://doi.org/10.1063/5.0027106

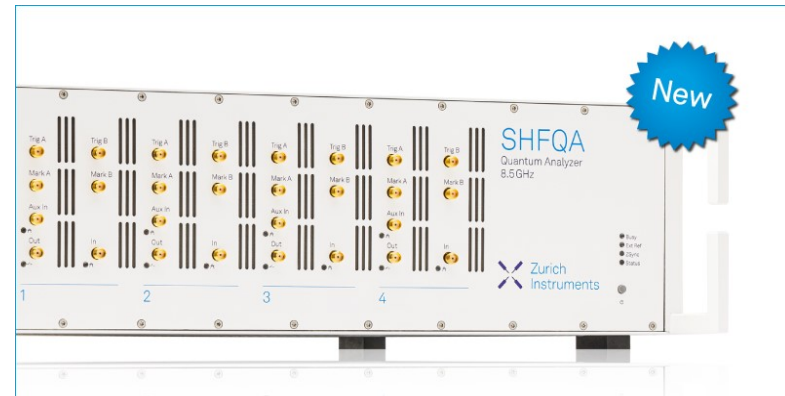

\section{Your Qubits \\ - Readout for up to 64 qubits \\ - Operation at up to $8.5 \mathrm{GHz}$ mixer-calibration-free \\ - Signal optimization with minimal latency}

Meet the next generation of quantum analyzers 


\title{
Effect of Diameter Ratio on Unsteady Flow Past Two Side- By-Side Cylinders at $\operatorname{Re}=\mathbf{2 0 0 0}$
}

\author{
R. Rajita Shenoi ${ }^{1, \mathrm{a}}$, Neeraj P. Maneli12,b ${ }^{2, \mathrm{~T}}$. Sundararajan ${ }^{3, \mathrm{c}}$ and Shaligram Tiwari ${ }^{4, \mathrm{~d}}$ \\ ${ }^{1}$ Maritime Technology Division, Ghent University, Ghent-9000, Belgium \\ 2,3,4 Department of Mechanical EngineeringIIT Madras, Chennai-600036, India \\ arajita.shenoi@gmail.com, ${ }^{b}$ neeri.89@gmail.com,ctsundar@iitm.ac.in, ${ }^{d}$ shaligt@iitm.ac.in
}

\begin{abstract}
Three-dimensional computations have been performed to study the effect of variation of cylinder size on flow characteristics of two cylinders in side-by-side arrangement. The RANS simulations are performed using the commercial solver, STAR-CCM $+{ }^{\circledR}$. Effect of variation of diameter ratio (DR) between two cylinders at fixed gap ratio and moderate Reynolds numbers $(\mathrm{Re}=2000)$ on hydrodynamic characteristics, such as drag and lift force coefficients as well as wake characteristics, such as Strouhal number $(\mathrm{St})$ has been presented. The coupled interactions between the cylinders in sideby-side arrangements are studied by means of cross-recurrence plots.
\end{abstract}

\section{INTRODUCTION}

Flow past cylindrical bodies is a classical research topic in hydrodynamics and has wide range of practical engineering applications. Estimation of forces around a structure is influenced by the flow around it and becomes important from design and safety perspective. The study of flow past multiple cylindrical bodies in different arrangements, namely, tandem, side-by-side and staggered is enough challenging due to complex interactions between cylinder wakes. A comprehensive review of experimental and numerical work of identical circular cylinders in different arrangements in the recent past has been described in Sumner [1]. Many researchers have performed numerical investigations to study the wake interactions between identical cylindrical bodies in side-byside arrangements [2] [3] [4].

Offshore engineering applications, such as piggyback pipelines, have multiple cylindrical arrangements with different diameters which are subjected to fluid flow. Gao et al.[5] performed 2-D simulations about two side-byside cylinders with different diameter ratio (DR) and gap ratio at Reynolds number, $\operatorname{Re}=300$ and concluded that the flow patterns of unequal cylinders had distinct in-phase and anti-phase vortex shedding with random switching of modes. Song et al. [6] used dye flow visualization and particle image velocimetry (PIV) to study the vortex interactions and flow structures behind side-by-side cylinders with DR $=2$ at different gap ratios and Re. The critical gap ratio was found to be smaller than that obtained with cylinders of equal diameter. Qiu et al.[7] performed computations using Large Eddy Simulations (LES) at subcritical $\mathrm{Re}=3900$ and $\mathrm{DR}=2$ for various gap ratios and observed that at a gap ratio of 1.2 the vortex shedding behind the main cylinder gets completely suppressed by that of control cylinder in side-by-side arrangement. From the survey of literature, it can be observed that study of flow past cylinders with identical diameter is widely researched upon when compared to cylinders with unequal diameters, especially in subcritical Re range. Present work investigates the viscous flow past multiple cylindrical bodies in side-by-side arrangement with different diameter ratios (DR) at $\mathrm{Re}=2000$ using numerical method. The gap ratio is fixed at 1.5 and DR varies in a range from 0.5 to 2 .

International Conference of Numerical Analysis and Applied Mathematics ICNAAM 2019

AIP Conf. Proc. 2293, 030027-1-030027-5; https://doi.org/10.1063/5.0028770

Published by AIP Publishing. 978-0-7354-4025-8/\$30.00 


\section{PROBLEM DEFINITION}

The computational domain adopted and a grid generated for numerical simulations of flow two circular cylinders in side-by-side arrangements is given in Fig. 1.

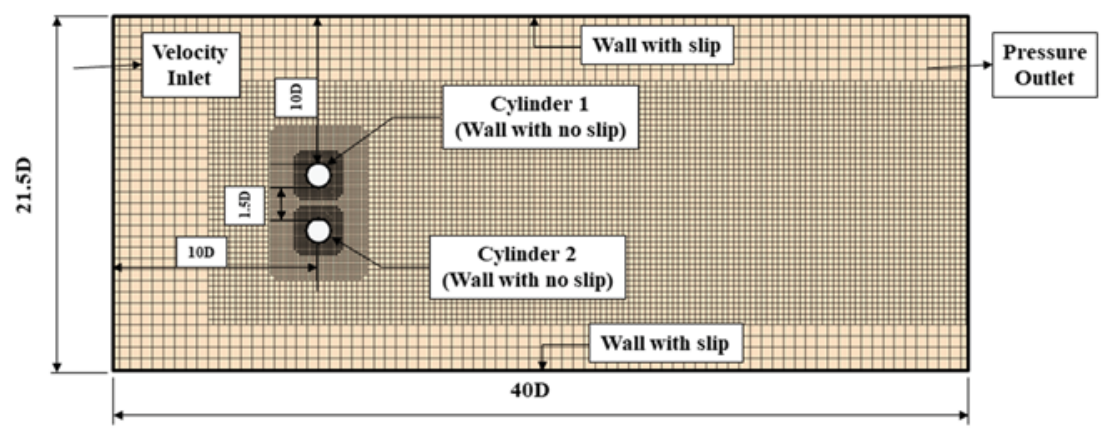

FIGURE 1. Computational domain and grid with boundary types specified

The grid shown here is that of the identical cylinder case. The span is taken as $4 \mathrm{D}$ for all simulations. The diameter of main cylinder ( cylinder 1) is taken as $0.1 \mathrm{~m}$ and the control cylinder (cylinder 2) changes with diameter ratio. Diameter ratio $(\mathrm{DR}=\mathrm{d} / \mathrm{D})$ is defined as ratio of diameter of cylinder 2 (d) to that of cylinder $1(\mathrm{D})$ and the chosen values are $0.5,0.75,1.0,1.25,1.5$ and 2.0. The gap ratio is defined as the spacing between cylinders to the diameter (D) of cylinder 2 and the value considered for present study is 1.5. All simulations are conducted at a moderate value of $R e=2000$ calculated based on the cylinder 1 . Unstructured trimmed hexahedral cells are used in the core of the domain while prismatic layers are employed near the wall with the help of preprocessor of STARCCM $+^{\circledR}$. In order to capture the flow characteristics around the cylinders as well as their wake, a separate volumetric block with finer grid is created. A thorough grid independence study is conducted and an optimum grid having 1.1 million cells is chosen for the simulations.

\section{NUMERICAL METHODOLOGY}

Unsteady Reynolds Averaged Navier-Stokes equations (URANS) employed in the present study are obtained by taking ensemble average of the instantaneous mass and momentum conservation equations for incompressible flows.

$$
\begin{gathered}
\frac{\partial \bar{u}_{i}}{\partial x_{i}}=0 \\
\rho\left[\frac{\partial \bar{u}_{i}}{\partial t}+\bar{u}_{k} \frac{\partial \bar{u}_{i}}{\partial x_{k}}\right]=-\frac{\partial p}{\partial x_{i}}+\frac{\partial}{\partial x_{j}}\left(v \frac{\partial \bar{u}_{i}}{\partial x_{j}}\right)-\frac{\partial\left(\overline{u_{i} u_{j}}\right)}{\partial x_{j}}
\end{gathered}
$$

where subscripts $i, j, k$ indicate respective components of Cartesian coordinate system. $\bar{u}$ and $\bar{p}$ represents time averaged velocity and pressure fields, $t$ represents time, $\rho$ represents density and $v$ is the kinematic viscosity. The last term in the right hand side of eqn. (2) is called Reynolds stress which is modelled using realisable $k-\varepsilon$ model proposed by Shih et al. [8]. Inlet is assigned as a velocity inlet with $u=U_{\infty}, v=0, w=0$ while outlet is assumed to be pressure outlet with $p=P_{\infty}$. Free shear boundary conditions are imposed on sides, top, and bottom walls. No-slip wall type boundary conditions are imposed on cylinder surfaces. The boundary values for turbulent kinetic energy, $k$ and turbulent dissipation rate, $\varepsilon$ are $0.001 \mathrm{Jkg}^{-1}$ and $0.1 \mathrm{~m}^{2} \mathrm{~s}^{-3}$, respectively.

The physical model and solver settings implemented for simulations are 3-D with unsteady, implicit, segregated solver. The pressure-velocity coupling is solved using SIMPLE (Semi-Implicit Method for Pressure Linked Equations) algorithm. A second order upwind scheme for spatial discretization together with second order temporal 
discretization has been used. A two equation realizable $k-\varepsilon$ turbulence model with low $\mathrm{y}+$ wall treatment is used to accurately capture the forces acting on the cylinder.

\section{RESULTS AND DISCUSSION}

The numerical model was validated by performing benchmark case of single stationary cylinder subjected to uniform flow at $\mathrm{Re}=3900$. Standard hydrodynamic quantities like mean drag coefficient $\left(\mathrm{Cd}_{\mathrm{m}}\right)$ and rms lift coefficient $\left(\mathrm{Cl}_{\text {rms }}\right)$ are calculated. Vortex shedding frequency $\left(f_{v}\right)$ is obtained from the power spectra of lift force fluctuations and Strouhal number $(\mathrm{St})$ is calculated as $\left(f_{v} D / U\right)$. The results are in good agreement with those from previously published studies [9] [10] and thus justify the present computational model.

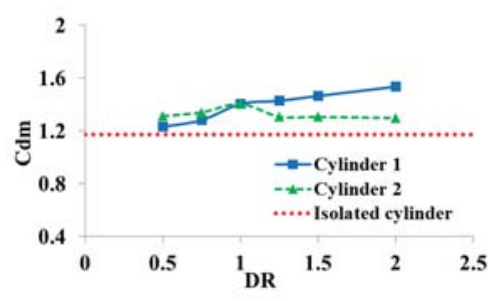

(a)

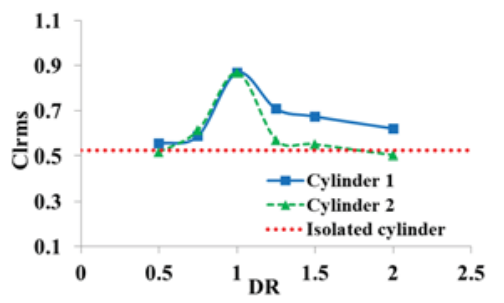

(b)

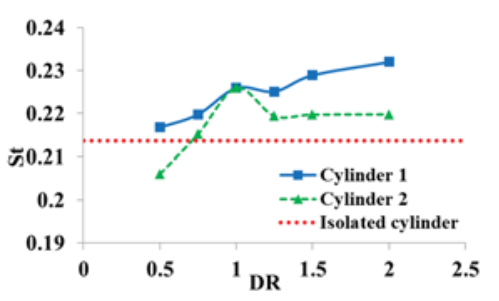

(c)

FIGURE 2. Variation of (a) mean drag coefficient $\left(\mathrm{Cd}_{\mathrm{m}}\right)$ and (b) rms lift coefficient $\left(\mathrm{Cl}_{\mathrm{rms}}\right)$ in the wake of cylinders with DR

The validated numerical model is used to perform simulations of flow around side-by-side cylinders and study the effect of DR on mean drag coefficient $\left(\mathrm{Cd}_{\mathrm{m}}\right)$, rms lift coefficient $\left(\mathrm{Cl}_{\mathrm{rms}}\right)$ and St. Figure 4(a), (b) and (c) shows variation of $\mathrm{Cd}_{\mathrm{m}}, \mathrm{Cl}_{\mathrm{rms}}$ and $\mathrm{St}$ of cylinders 1 and 2 with different $\mathrm{DR}$, respectively. Results of isolated cylinder case at $\operatorname{Re}=2000$ are also included in the graphs for comparison. It can be clearly seen that the inclusion of a control cylinder in the side-by-side cylinder arrangement has significant influence on the forces of the main cylinder. It can be observed for the main cylinder or cylinder $1, \mathrm{Cd}_{\mathrm{m}}$ increases with increase in DR. However, $\mathrm{Cl}_{\mathrm{rms}}$ increases with increase in DR till it takes the value of 1.0 after which it gradually drops down with DR $>1$. Similar trend is observed for cylinder 2 as well. In case of St, the value increases with increase in DR except for DR $=1.25$ for cylinder 1 . For cylinder 2, St takes a steep increase till $\mathrm{DR}=1$ after which it drops to a lower value at $\mathrm{DR}=1.25$ and remains without much increase as seen in case of cylinder 1. 

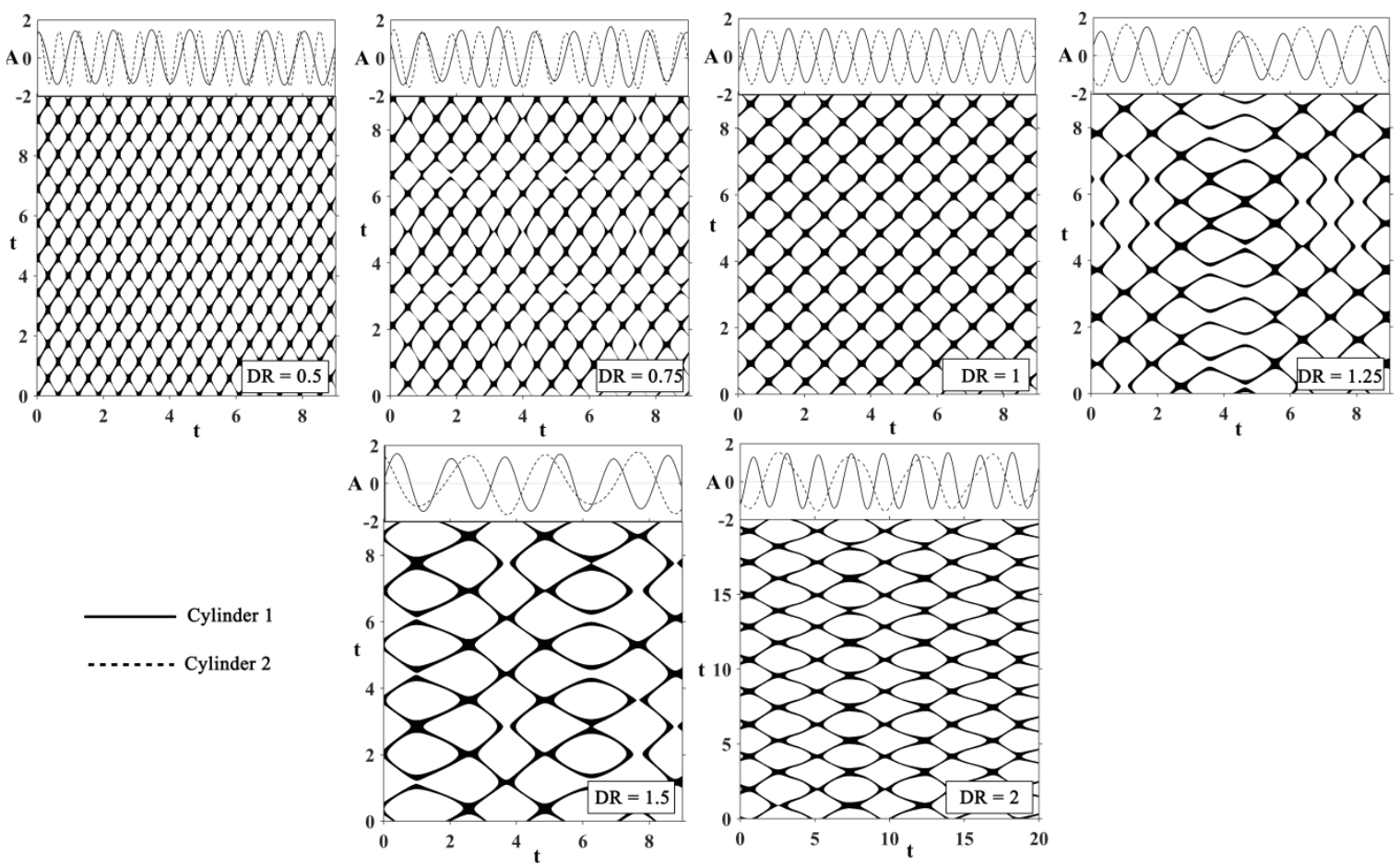

FIGURE 3. Cross-recurrence plots of lift signals of side-by-side cylinders for different DR values at GR = 1.5

Recurrence plot is a powerful tool to study the dynamic behavior of system in reconstructed phase space. The methodology as well as practical application of recurrence plots and analysis is well described in Marwan et al. [11]. The nonlinear interactions and dependency between two dynamic systems (two cylinders) can be studied by means of cross-recurrence plots (CRP). Figure 3 depicts the CRP between time history of lift forces of cylinders 1 and 2, for different values of $\mathrm{DR}$ at $\mathrm{GR}=1.5$. The dark regions in the CRP indicate the recurrence of one system over the other. At $\mathrm{DR}=0.5$ and 1 , well-ordered patterns with straight diagonals represent strong synchronization between cylinder wakes. The bowed lines observed in CRP for DR $=0.75$ and 2 indicate mismatch between wavelengths. The dislocation indicated in CRP for $\mathrm{DR}=1.25$ and 1.5 shows a time shift between the two signals. The results of CRP can be quantified by performing the recurrence quantification analysis to give better insights into the coupled response of the signals. This forms the future work of the present study.

\section{CONCLUSION}

The influence of varying diameter ratio on unsteady wake characteristics around two circular cylinder in side-byside arrangement at $\mathrm{Re}=2000$ is brought out from present study. Computations are performed on flow past cylinders at different DR $(0.5,0.75,1,1.25,1.5,2)$ and fixed spacing between them $(1.5 \mathrm{D})$. From the validation study, it can be concluded that RANS based CFD is capable of predicting the flow characteristics satisfactorily for computations in moderate Re range. The inclusion of control cylinder influences the forces on the main cylinder. With increase in DR, the mean drag coefficient, $\mathrm{Cd}_{\mathrm{m}}$ of main cylinder increases. The nonlinear interactions between cylinder wakes are brought out by performing cross recurrence analysis. This analysis gives deeper insights on synchronization characteristics between cylinder wakes. The work can be further extended to study the effect of gap ratio and propose an optimal arrangement of side-by-side cylinder configuration at $R e=2000$. 


\section{REFERENCES}

1. D. Sumner, Two circular cylinders in cross flow: A review, Journal of Fluids and Structures, 26 (2010) 849-899.

2. L. Chena, J.Y. Tub, G.H. Yeoh, Numerical simulation of turbulent wake flows behind two side-by-side cylinders, Journal of Fluids and Structures, 18 (2003) 387-403.

3. N. Kondo, D. Matsukuma, Three-dimensional computation for flow around two circular cylinders in side-byside arrangement by a third-order upwind finite element method, International Journal of Computational Fluid Dynamics, 19:1(2005), 37-43.

4. I. Afgan, Y. Kahil, S. Benhamadouche, P. Sagaut, Large eddy simulation of the flow around single and two sideby-side cylinders at subcritical Reynolds numbers, Physics of Fluids 23(2011), 075101.

5. Y.Y. Gao, X.K.Wang, S.K.Tan, Numerical study of two side-by-side cylinders with unequal diameters at low Re, $26^{\text {th }}$ IAHR Symposium on Hydraulic Machinery and systems, 15(2012) 062041.

6. F.L. Song, S. Y. Tseng, S. W. Hsu, C. H. Kuo, Gap ratio effect on flow characteristics behind side-by-side cylinders of diameter ratio two. Experimental Thermal and Fluid Science, 66, (2015), 254-268.

7. X. Qiu, Z-X. Bi, J-P. Luo, Y-L. Liu, Vortex shedding in the flow around two side-by-side circular cylinders of different diameters, Journal of Hydrodynamics, 29:3 (2017): 470-478.

8. T.-H. Shih, W. W. Liou, A. Shabbir, Z. Yang, J. Zhu, A New k- Eddy Viscosity Model for High Reynolds Number Turbulent Flows -- Model Development and Validation, NASA TM, (1994)106721.

9. M. Breuer, Large Eddy Simulation of the subcritical flow past a circular cylinder: numerical and modeling aspects, International Journal for Numerical Methods in Fluids, 28(1998), 1280-1302.

10. M. Prsic, M. C. Ong, B. Pettersen, D. Myrhaug, Large Eddy Simulations of three-dimensional flow around a pipeline in a uniform current", 31st International Conference on Ocean Offshore and Artic Engineering OMAE, July 1-6, 2012, Rio de Janeiro, Brazil.2012.

11. N. Marwan, M. C. Romano, M. Thiel, J. Kurths, Recurrence plots for the analysis of complex systems. Physics reports, 438(5-6),(2007), 237-329. 